\title{
Quetiapine Related Acute Paralytic Ileus in a Bipolar I Disorder Patient with Successful Low Dose Amisulpride Substitution: A Case Report
}

\author{
Shun-Ting Chiang, Chen-Chia Lan \\ Department of Psychiatry, Taichung Veterans General Hospital, Taichung, Taiwan
}

\begin{abstract}
The mechanism of medication-induced gastrointestinal hypomotility is primarily caused by muscarinic cholinergic antagonism. This effect may cause constipation and paralytic ileus, which may lead to fatal complications. A 51-year-old woman was admitted due to manic episode recurrence. She developed paralytic ileus under quetiapine use and treated successfully under low dose amisulpride use. The related mechanism, associated risk factors, and the rationale for medication switch are discussed.
\end{abstract}

KEY WORDS: Bipolar disorder; Paralytic ileus; Constipation; Quetiapine; Amisulpride; Anticholinergics.

\section{INTRODUCTION}

The mechanism of medication-induced gastrointestinal hypomotility is primarily caused by muscarinic cholinergic antagonism. This effect may cause constipation and paralytic ileus, which may lead to fatal complications. Different antipsychotics have different anticholinergic potencies, which should be taken into consideration during medication selection. In this report, we discuss a bipolar I disorder patient who suffered from paralytic ileus and successfully treated without further complication after medication switch to amisulpride use.

\section{CASE}

A 51-year-old woman was previously healthy and without personal history of any psychiatric or medical illnesses. Her older sister has bipolar disorder. She has been a pious adherent of Buddhism for more than 15

Received: December 13, 2016/Revised: January 6, 2017 Accepted: January 13, 2017

Address for correspondence: Chen-Chia Lan, MD

Department of Psychiatry, Taichung Veterans General Hospital,

1650 Taiwan Boulevard Sect. 4, Taichung 40705, Taiwan

Tel: +886-4-23592525, Fax: +886-4-23595046

E-mail: drblue3305@gmail.com

ORCID: https://orcid.org/0000-0003-3264-6183 years.

In June 2015, she was sent to the emergency department due to irritable mood, decreased need for sleep, hypertalkativity, flight of ideas, grandiose delusion and auditory hallucination for 1 week. Serial examinations including brain computed tomography and thyroid function tests showed no abnormal findings. Under the impression of bipolar I disorder, manic episode with psychotic features, admission was suggested by the consulting psychiatrist but patient refused due to poor insight. Compulsive admission was not indicated due to no obvious risks of self-harm or violence at that time.

She was discharged against advice from the emergency department but soon admitted to another hospital due to persistent manic symptoms. However, due to poor insight, she was discharged against medical advice after only 5 days of treatment and with minimal improvement. She discontinued all medication and refused to visit the outpatient clinic after discharge.

This manic episode eventually resolved 3 months later. Unfortunately, she was found to have elevated mood, decreased need for sleep, hypertalkativity, flight of ideas, grandiose delusion and some disturbing behavior during religious practice for more than 2 weeks in May 2016. She was sent to our hospital again and admission was arranged under the impression of bipolar I disorder, manic

(c) This is an Open-Access article distributed under the terms of the Creative Commons Attribution Non-Commercial License (http://creativecommons.org/licenses/by-nc/4.0) which permits unrestricted non-commercial use, distribution, and reproduction in any medium, provided the original work is properly cited. 

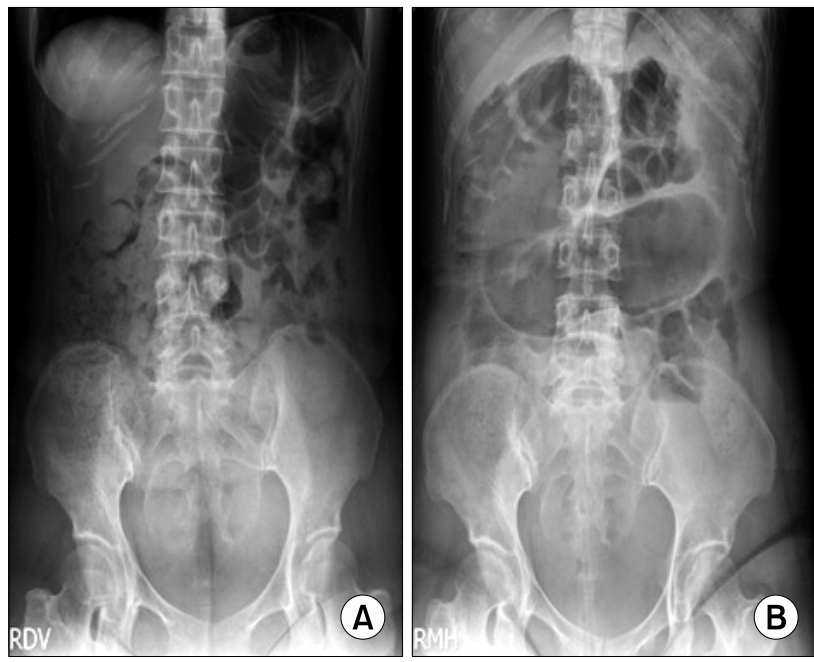

episode with psychotic features.

Serial examinations including thyroid function tests were done and no abnormal finding was noted on day 1 . We delivered quetiapine $400 \mathrm{mg} /$ day and valproic acid $1,000 \mathrm{mg} /$ day for controlling her manic and psychotic symptoms. We did not use any anticholinergic medication in the entire treatment due to the absence of extrapyramidal symptoms. The valproic acid blood level was $88.94 \mu \mathrm{g} / \mathrm{ml}$ on day 10 . Elevated mood was improving with reduced frequency of disturbing behaviors at ward.

On day 15 , she complained about abdominal distention and constipation. A kidney, ureter, and bladder (KUB) X-ray revealed focal dilated loops suspecting focal obstruction (Fig. 1A). We delivered cathartics and prokinetic agents with sennoside and domperidone. Sodium phosphate monobasic enema was later performed twice due to limited stool passage. However, aggravated abdominal distention, anorexia, and general weakness were noted on day 17 . We tried to taper down quetiapine to $200 \mathrm{mg} /$ day and continued valproic acid use. However, severe abdominal pain and urine retention were found at night on day 17. KUB X-ray revealed gaseous dilatation of the transverse colon and much fecal material retention in colon (Fig. 1B). Nothing by mouth except medication was implemented and decompression with both the anal tube and the nasogastric tube were performed. Quetiapine was also discontinued immediately. The markedly abnormal laboratory findings were leukocytosis with left shift (white blood cells, 10,070/ $\mu$; neutrophil, 76\%) and a newly-developed hypothyroidism (free thyroxine [T4]: $5.48 \mathrm{pg} / \mathrm{ml}$, thyroid-stimulating hormone [TSH]: $3.09 \mu \mathrm{lU} / \mathrm{ml}$ on day

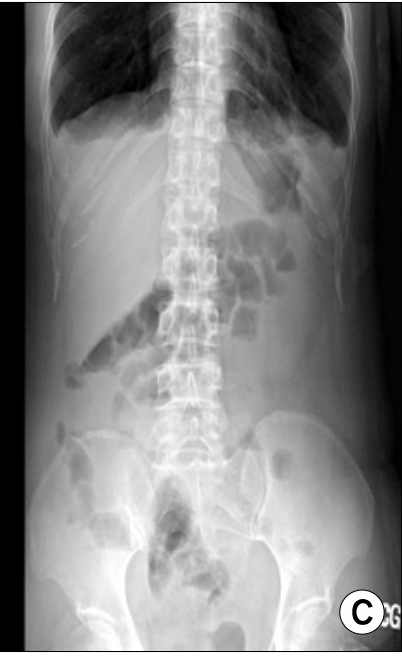

Fig. 1. The serial kidney, ureter, and bladder (KUB) X-rays of the patient showing dilated focal loops on day $10(\mathrm{~A})$; worsening of ileus with significantly gaseous distension of the transverse colon and retention of fecal materials on day 17 (B); and the improvement of paralytic ileus on day 22 (C).
17; compared to free T4: $11.00 \mathrm{pg} / \mathrm{ml}, \mathrm{TSH}: 1.86 \mu \mathrm{lU} / \mathrm{ml}$ on day 1). Antithyroglobulin antibody and anti-thyroid peroxidase antibody levels were normal, which revealed no evidence of autoimmune related thyroid disease. Gastroenterology consultation suggested quetiapine related paralytic ileus superimposed with hypothyroidism. Endocrinology consultation also suspected quetiapine related hypothyroidism. Both consultations agreed with our interventions and recommended further observation of her paralytic ileus and thyroid function.

After quetiapine was withheld, her paralytic ileus improved significantly with fair oral intake and stool passage observed the next day. However, manic symptoms with irritable mood and hypertalkativity exacerbated 2 days after the discontinuation of quetiapine despite the continuous use of valproic acid. We then added amisulpride to $400 \mathrm{mg} /$ day on day 22. KUB X-ray follow-up demonstrated obvious improvement of the paralytic ileus (Fig. 1C). She tolerated well with amisulpride $400 \mathrm{mg} /$ day and valproic acid 1,000 mg/day use and her manic symptoms subsided gradually. We rechecked her thyroid function which revealed improving hypothyroidism state on day 30 (free T4: $7.81 \mathrm{pg} / \mathrm{ml}$, TSH: $3.86 \mu \mathrm{lU} / \mathrm{ml}$ ). She was later successfully discharged on day 31 under stable condition both mentally and physically.

\section{DISCUSSION}

The mechanism of medication-induced gastrointestinal hypomotility is primarily muscarinic cholinergic antagonism. ${ }^{1}$ Previous study revealed that increasing age, fe- 
male gender, treatment with clozapine, high potency first generation antipsychotics, tricyclic antidepressants, anticholinergics and opioids were associated with an increased risk of ileus." One systematic review study recently found history of abdominal surgery, longer duration of psychiatric disorders, and older age to be risk factors of relapse of ileus. ${ }^{2)}$ Overall, patients treated with antipsychotics had a 1.9 times higher risk to develop constipation compared to non-users. In severe cases, constipation may progress to ileus and bowel ischemia with multiple fatalities related to sepsis and bowel perforation. ${ }^{3)}$ Therefore, we should pay more attention to antipsychotic-related constipation and further complications, such as ileus, to prevent possible fatalities.

Anticholinergic potency differs markedly among antipsychotics. ${ }^{1)}$ Among atypical antipsychotics, clozapine, olanzapine, and quetiapine have significant affinity for the muscarinic receptors in vitro, while aripiprazole, risperidone, and ziprasidone do not. ${ }^{4)}$ Clozapine has the highest risk for anticholinergic related gastrointestinal adverse effect. ${ }^{1)}$ One report revealed a patient died from fecal impaction and associated aspiration of feculent vomitus and another patient developed fatal bowel ischemia within 2 days. ${ }^{1)}$ Quetiapine was also prone to cause anticholinergic side effects. In the Clinical Antipsychotic Trials of Intervention Effectiveness (CATIE) study, quetiapine was found to have significantly more anticholinergic side effects in phase 1, phase2, and phase 3 trials. ${ }^{5)}$ Anticholinergic adverse effects had been found in $27 \%$ of the patients treated with quetiapine, and the most common complaints were dry mouth, urinary hesitancy and constipation. ${ }^{5)}$ Of note, our patient was antipsychotic-free for 9 months prior to this admission and developed paralytic ileus within 15 days after quetiapine use, which was clearly different from the average onset of 3 years after first antipsychotic prescription reported by Nielsen and Meyer. ${ }^{1)}$ However, another case of acute paralytic ileus within 8 days of risperidone use has also been reported. ${ }^{6}$ Thus, acute onset paralytic ileus related to antipsychotic use is possible and we should be aware of this and manage it promptly if encountered.

As for amisulpride, it is a serotonin dopamine antagonist known for its properties of predominantly pre-synaptic D2/D3 dopamine receptor binding and partial D2 agonism. ${ }^{7)}$ Although not recommended and US Food and Drug Administration-approved as standard bipolar mania treatment, it did show good response rate for acute mania compared to haloperidol in adjunct to divalproex therapy. ${ }^{8)}$ Amisulpride monotherapy use in one patients with bipolar I disorder also showed there may be some benefits for maintenance treatment of bipolar disorder. ${ }^{9}$ Significant decrease in overall relapse rates, especially manic episodes, by amisulpride treatment was also found in one previous study. ${ }^{10)}$ In addition, no significant anticholinergic properties was associated with amisulpride and it only possessed minimal risk for constipation compared with other atypical antipsychotics. ${ }^{1)}$ These were the main reasons we substituted quetiapine with amisulpride to control the patient's manic symptoms and minimized anticholinergic-related gastrointestinal hypomotility.

The other point worthy of discussion is the relationship between anticholinergics, hypothyroidism, and the paralytic ileus in our patient. Gastrointestinal hypomotility was known to be associated with hypothyroidism and a few cases of paralytic ileus associated with hypothyroidism were reported. ${ }^{11)}$ Furthermore, hypothyroidism was reported to be an adverse effect of antipsychotics. Previous study has shown that $0.4 \%$ treated with quetiapine experienced TSH elevations and $60 \%$ among them needed thyroid hormone supplementation. ${ }^{12)}$ Therefore, there is a possibility that other than cholinergic antagonism, quetiapine may cause paralytic ileus via influencing thyroid function. However, the logical management would still be discontinuing quetiapine to reduce its adverse effect on the thyroid gland. Although there is also a possibility that amisulpride may induce hypothyroidism, currently only case report has been published. ${ }^{13)}$ And the improvement of the hypothyroid state in our patient after switching from quetiapine to amisulpride did not support the idea of amisulpride-related hypothyroidism. Currently, there is insufficient literature determining the potential of inducing hypothyroidism by various antipsychotics and further studies are needed in order to guide antipsychotics use in patients with thyroid dysfunctions. ${ }^{14)}$

Considering the prevalence of quetiapine related anticholinergic side effects and quetiapine related hypothyroidism, we suggested that the focus should still be on reducing cholinergic antagonism by discontinuing or switching antipsychotics when managing paralytic ileus in bipolar patients to prevent potential fatalities. From the treatment experience of our patient, amisulpride was a rationale and successful substitute for quetiapine as it was 
well tolerated without anticholinergic side effects and showed good response in treating her manic episode.

\section{REFERENCES}

1. Nielsen J, Meyer JM. Risk factors for ileus in patients with schizophrenia. Schizophr Bull 2012;38:592-598.

2. Kitahata R, Nakajima S, Suzuki T, Plitman E, Mimura M, Uchida $\mathrm{H}$. Relapse of ileus in patients with psychiatric disorders: A 2-year chart review. Gen Hosp Psychiatry 2016;38: 31-36.

3. De Hert M, Dockx L, Bernagie C, Peuskens B, Sweers K, Leucht $\mathrm{S}$, et al. Prevalence and severity of antipsychotic related constipation in patients with schizophrenia: a retrospective descriptive study. BMC Gastroenterol 2011;11:17.

4. Chew ML, Mulsant BH, Pollock BG, Lehman ME, Greenspan A, Kirshner MA, et al. A model of anticholinergic activity of atypical antipsychotic medications. Schizophr Res 2006;88: 63-72.

5. De Hert M, Hudyana H, Dockx L, Bernagie C, Sweers K, Tack J, et al. Second-generation antipsychotics and constipation: a review of the literature. Eur Psychiatry 2011;26:34-44.

6. Ramamourthy P, Kumaran A, Kattimani S. Risperidone associated paralytic ileus in schizophrenia. Indian J Psychol Med 2013;35:87-88.

7. Perrault G, Depoortere R, Morel E, Sanger DJ, Scatton B. Psychopharmacological profile of amisulpride: an antipsy- chotic drug with presynaptic D2/D3 dopamine receptor antagonist activity and limbic selectivity. J Pharmacol Exp Ther 1997;280:73-82.

8. Thomas P, Vieta E. Amisulpride plus valproate vs haloperidol plus valproate in the treatment of acute mania of bipolar I patients: a multicenter, open-label, randomized, comparative trial. Neuropsychiatr Dis Treat 2008;4:675-686.

9. Hong CC, Chen CK, Yeh TC, Chu CS, Chen TY. Amisulpride monotherapy may be a choice of maintenance treatment for patients with both bipolar / disorder and metabolic syndrome. Aust N ZJ Psychiatry 2015:49:757-758.

10. Carta MG, Zairo F, Mellino G, Hardoy MC, Vieta E. An open label follow-up study on amisulpride in the add-on treatment of bipolar I patients. Clin Pract Epidemiol Ment Health 2006;2:19.

11. Rodrigo C, Gamakaranage CS, Epa DS, Gnanathasan A, Rajapakse S. Hypothyroidism causing paralytic ileus and acute kidney injury - case report. Thyroid Res 2011;4:7.

12. Ramaswamy S, Siddiqui Z, Saharan S, Gabel TL, Bhatia SC. Quetiapine-induced hypothyroidism. I Psychiatry Neurosci 2005;30:57.

13. Mendhekar DN, Andrade C. Amisulpride-induced tardive dyskinesia and possible association of dyskinesia with hypothyroidism. J Neuropsychiatry Clin Neurosci 2009;21:104-105.

14. Bou Khalil R, Richa S. Thyroid adverse effects of psychotropic drugs: a review. Clin Neuropharmacol 2011;34:248-255. 